\title{
comparative analysis of power-law type fin problem using wavelet collocation and Galerkin methods
}

\author{
Surjan Singh ${ }^{1 *}$, Subrahamanyam Upadhyay ${ }^{1}$, K. N. Rai ${ }^{2}$ \\ 1 DST-CIMS, Banaras Hindu University, Varanasi-221005, U.P. India \\ 2 Department of Mathematical Sciences, IIT BHU Varanasi 221005 India \\ *Corresponding author E-mail: surjan.singhbhu@gmail.com
}

\begin{abstract}
Copyright (C)2014 Surjan Singh et.al. This is an open access article distributed under the Creative Commons Attribution License, which permits unrestricted use, distribution, and reproduction in any medium, provided the original work is properly cited.
\end{abstract}

\begin{abstract}
In this paper, Wavelet Collocation Method and Wavelet Galerkin Method have been used to evaluate the temperature distribution of a straight rectangular fin. The linear problem has been solved by Wavelet Galerkin Method while non-linear problem by Wavelet Collocation Method. It has been observed that accuracy increases as the number of basis function increases. The result thus obtained is compared with other available results obtained by using approximate analytic methods such as Adomian Decomposition Method, Differential Transformation Method as well as exact solution. It has been observed that the result obtained by present method is exactly same as that obtained by exact method. The method provides a unique solution for $n=-3 / 2, N= \pm 0.9$ and $n=-3, N= \pm 0.4$. The justification of unique solution gets confirmed from Figs. 7 and 8 . The present method provides single solution for all existing values. The convergence analysis of the proposed method along side numerical procedure for this boundary value problem is given to test wider applicability and accuracy of the method.
\end{abstract}

Keywords: linear, non-linear, differential equation, fins, wavelet Galerkin, collocation method.

\section{Introduction}

Fins are generally used for heat exchange purpose. Examples of fin are radiator in vehicles and heat exchangers in power plants. In electrical devices like motors and transformers, the generated heat can be efficiently transferred. Other applications are petrochemical plants, gas treatment plants, natural gas liquefaction plants, air separation plants, helium liquefaction plants, etc. In the study of heat transfer, fin is a surface, made by metallic material which is used to increase the rate of heat transfer to environment. The rate of heat transfer depends on the surface area of the fin. In nature the ears of rabbit act as fins to release heat from the blood. In a series of papers, Chiu and Chen (2002) used Adomian decomposition method to solve the fin problem and evaluated the efficiency for different number of terms. In that paper non-linear problem were treated as linear problem. Rai and Rai (2003) used finite difference method and obtained results for different order polynomials. Kim and Hung (2007) obtained series solution for non-linear fin problem and assumed that the thermal conductivity is the linear function of temperature. Chang (2005) considered straight fin and defined heat transfer coefficient $h$ in terms of the power-law-type dependence on the local temperature difference between the fin and ambient fluid. Ganji et al. (2007) used Homotopy Perturbation Method (HPM), Variational Iteration Method (VIM) and perturbation method and concluded 
that the results obtained by VIM and HPM converged faster than those obtained by the perturbation method; moreover, HPM was faster than (VIM). Dul'kin and Garas'co (2002) (2008), studied the analytical solution of one dimensional heat conduction problem for a single fin with temperature dependent heat transfer. Arslanturk (2005), Rajabi (2007), and domairry and Fazeli (2009) solved same problem by Homotopy perturbation method, Adomian decomposition method and obtained good results.

Cortel (2008) used numerical analysis technique for solution of fin problem and obtained results for different values of $N$ and $n$. Chowdhury et al. (2009) compared homotopy analysis method with homotopy-perturbation method and Adomian decomposition method. HPM and ADM are special cases of the HAM. Khaini et al.(2009) considered a straight one-dimensional fin and used homotopy analysis method to evaluate the analytical approximate solution. Efficiency of fin with temperature dependent thermal conductivity was obtained as a function of thermo-geometric fin parameter. Moradi (2011) used Differential Transformation Method (DTM) and compared result with Adomian Decomposition Method (ADM). Abbasbandy and Shivanian (2010) found exact analytical solution in implicit form. Momoniat (2012) obtained fin efficiency based on Newton's law of cooling. He showed that the second formula for efficiency mentioned in his paper was exact when the first integral could be determined. Razzaghi and yousefi (2001) presented an operational matrix of integration based on Legendre wavelets and solved initial value problem. Mohammadi et al. (2011) used Legendre wavelets Galerkin method for solving ordinary differential equations with non-analytic solution. Frazier (1999) used wavelet Galerkin method in order to solve two point boundary value problem of ordinary differential equation.

In the present study, an attempt has been made to solve linear and nonlinear boundary value problem of heat transfer in fin by using wavelet collocation and wavelet Galerkin methods. The paper is organized in the following way: In section 2 formulation of the problem is given. In section 3 Legendre wavelets are introduced and a general formulation of the WCM and WGM is presented. In section 4 algorithm is given. In section 5 brief convergence analysis is given. In section 6 verification of the method is performed and problem is discussed in detail. Section 7 represents concluding remarks and future research.

\section{Formulation of the problem}

Basic assumptions for heat transfer through a fin include the following: the temperature varies in one direction only; the material of fin is homogeneous; the temperature remains constant with time (steady state condition); the thermal conductivity is constant; there is no energy source within the fin; and temperature of surrounding medium is uniform. To determine heat transfer through a fin, the temperature distribution through the fin is needed. We consider a straight rectangular fin of length $L$ with a uniform cross section area $A c$. The circumferential parameter is $P$; thermal conductivity of fin material is $k$. The local heat transfer coefficient along with the fin height was considered to be exhibited as a power-law type dependence on the local temperature difference between the fin surface and the environment i.e. $h=h_{b}\left(\frac{T-T_{a}}{T_{b}-T_{a}}\right)^{n}$, where $T_{a}$ is the environment (ambient) temperature, $T$ is the local temperature on the fin surface, and $n$ depends on heat transfer mode. The value of $n$ can vary in a wide range depending on the mode of boiling. For practical purpose, the physical values of $n$ are $5 / 4$ and $4 / 3$ for free convection, 3 for nucleate boiling and 4 for radiation.

Energy balance equation in one dimensional steady state can be defined as

$k A c \frac{d^{2} T}{d x^{2}}-P h\left(T-T_{a}\right)=0$,

with boundary condition

$x=0, \frac{d T}{d x}=0$

$x=L, T=T_{b}$.

Using dimensionless and similarity criteria

$\theta=\frac{T-T_{a}}{T_{b}-T_{a}}, X=\frac{x}{L}, N=\sqrt{\frac{h_{b} P L^{2}}{k A c}}$. 
For one dimensional steady state the heat conduction equation in dimensionless form can be written as follows:

$$
\frac{d^{2} \theta}{d X^{2}}-N^{2} \theta^{n+1}=0
$$

Assume the fin tip is insulated and the boundary conditions subjected to equation (4) are

$\frac{d \theta(0)}{d X}=0$

$\theta(1)=1$

\section{Solution of the problem}

We solve this problem using Wavelet Collocation Method and Wavelet Galerkin Method

Wavelets: Continuous Wavelets defined by the following formula

$\psi_{a, b}(X)=|a|^{\frac{-1}{2}} \psi\left(\frac{X-b}{a}\right), a, b \in R, a \neq 0$,

where $\mathrm{a}$ is dilation parameter and $\mathrm{b}$ is a translation parameter

Legendre wavelets $\psi_{m, n}(X)=\psi(k, \hat{n}, m, X)$, where $\hat{n}=2 n-1, n=1,2, \ldots, 2^{k-1}, k$ is any positive integer, $m$ is the order of Legendre polynomials. Legendre wavelets defined on the interval $(0,1)$ by

$\psi_{m, n}(X)= \begin{cases}\sqrt{(m+1 / 2)} 2^{k / 2} P_{m}\left(2^{k} X-\hat{n}\right) & , \quad \frac{\hat{n}-1}{2^{k}} \leq X \leq \frac{\hat{n}-1}{2^{k}} \\ 0 & , \quad \text { otherwise }\end{cases}$

where $m=0,1, \ldots, M-1$ and $n=1,2, \ldots, 2^{k-1}$. Here $P_{m}(X)$ is the well known Legendre polynomials of order $\mathrm{m}$.

$P_{0}(X)=1, P_{1}(X)=X, P_{m+1}(X)=\frac{2 m+1}{m+1} X P_{m}(X)-\frac{m}{m+1} P_{m-1}(X)$,
$m=1,2,3, \ldots, M-1$.

A function $\mathrm{f}(\mathrm{X})$ defined in domain $[0,1]$ can be expressed as

$f(X)=\sum_{n=1}^{\infty} \sum_{m=0}^{\infty} c_{n, m} \psi_{m, n}(X)$,

where $c_{n, m}=<f(X), \psi_{m, n}(X)>$ in which $<., .>$ denotes the inner product. If we take some terms of infinite series, then (10) can be written as

$f(X)=\sum_{n=1}^{2^{k-1}} \sum_{m=0}^{M-1} c_{n, m} \psi_{m, n}(X)=C^{T} \psi(X)$,

where $\mathrm{C}$ and $\psi(X)$ are $2^{k-1} M \times 1$ Matrices given by

$$
\begin{gathered}
C=\left[c_{10}, c_{11}, \ldots, c_{1 M-1}, c_{20}, c_{21}, \ldots, c_{2 M-1}, c_{2^{k-1} 0} c_{2^{k-1} 1}, \ldots, c_{2^{k-1} M-1}\right]^{T} \\
\psi(X)=\left[\psi_{10}(X), \psi_{11}(X), \ldots, \psi_{1 M-1}(X), \psi_{20}(X), \ldots, \psi_{2 M-1}(X), \ldots\right. \\
\left.\psi_{2^{k-1} 0}(X) \psi_{2^{k-1} 1}(X), \ldots, \psi_{2^{k-1} M-1}(X)\right]^{T}
\end{gathered}
$$

\subsection{Property of the product of two Legendre wavelets}

If $\mathrm{E}$ is a given wavelets vector then we have the property

$E^{T} \psi \psi^{T}=\psi^{T} \hat{E}$

where $\hat{E}$ is $2^{k-1} M \times 2^{k-1} M$ matrices depend on the wavelet vector $\mathrm{E}$ 


\subsection{Operational matrix of integration}

The integration of the wavelets $\psi(X)$ which is defined in (8) can be obtained as ( Razzaghi and Yousefi 2001)

$\int_{0}^{X} \psi(s) d s=P \psi(X), X \in[0,1]$,

where $\mathrm{P}$ is $2^{k-1} M \times 2^{k-1} M$ operational matrix of integration given by

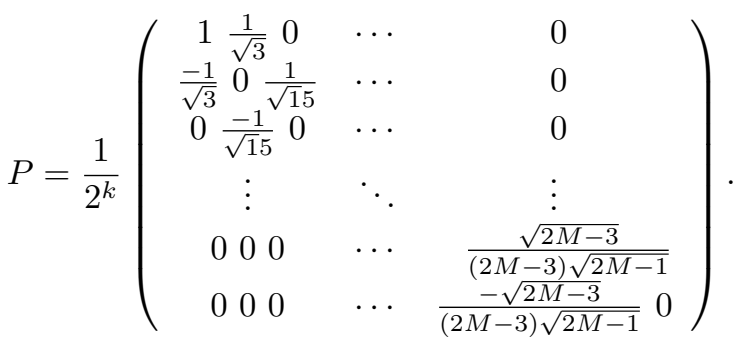

\subsection{Wavelet Colloaction Method}

Let

$\theta^{\prime \prime}(X)=C^{T} \psi(X)$

Integrating from 0 to $\mathrm{X}$, we get

$\theta^{\prime}(X)=C^{T} P \psi(X)+\theta^{\prime}(0)$

Again integrating above equation and using boundary conditions (5) and (6), we get

$\theta(X)=C^{T} P^{2} \psi(X)+d^{T} \psi(X)-C^{T} P^{2} \psi(1) d^{T} \psi(X)$

Substituting the value of $\theta(X)$ and $\theta^{\prime \prime}(X)$ in equation(4), we obtain

$C^{T} \psi(X)-N^{2}\left(C^{T} P^{2} \psi(X)+d^{T} \psi(X)-C^{T} P^{2} \psi(1) d^{T} \psi(X)\right)^{n+1}=R\left(X, c_{1}, c_{2}, \ldots, c_{n}\right)$

As $\theta(X)$ is an approximate solution of system (4) to (6). Choosing $n$ collocation points $X_{i}, i=1,2,3, \ldots, n$ in the interval $(0,1)$, at which residual $R\left(X, c_{1}, c_{2}, \ldots, c_{n}\right)$ equal to zero. The number of such points must be equal to the number of coefficients $c_{i}, i=1,2,3, \ldots, n$. Thus, we get $R\left(X, c_{1}, c_{2}, \ldots, c_{n}\right)=0, i=1,2,3, \ldots, n$.

Solving this system of equations using Newton-Raphson method, the coefficients $c_{i}, i=1,2,3, \ldots, n$ are evaluated and correspondingly temperature can be determined from equation (19).

\subsection{Wavelet Galerkin Method}

Putting $n=0$ in (20) and taking transpose, we get,

$\psi^{T}(X) C-N^{2}\left\{\psi^{T}(X) P^{2 T} C+\psi^{T}(X) d-\psi^{T}(X) d \psi^{T}(1) P^{2 T} C\right\}=0$

Removing $\psi^{T}(X)$ from left, we obtain

$\left(I-N^{2} P^{2 T}+N^{2} d \psi^{T}(1) P^{2} T\right) C=N^{2} d$.

Solving the above system of linear equations, we obtain value of $C$. Substituting the value of $C$ in equation (19), we obtain temperature $\theta$.

For $n=0$, the exact solution of (4) to (6) can be put in the form

$\theta=\frac{\cosh (N X)}{\cosh (N)}$ 


\section{Algorithm (MATLAB Programming procedure)}

Step1 Define all the constant values

Step 2 Define P, d, $\psi(x), \psi(1)$

Step $3 \mathrm{C}=[\mathrm{c} 1, \mathrm{c} 2, \ldots, \mathrm{c} 9]$

Step 4 Define $\mathrm{F}=$ transpose $(C) * S i-N^{2} *\left(1+\text { transpose }(C) * P^{2} *(S i-S i 1)\right)^{(n+1)}$

Step 5 Using collocation points as $\mathrm{X}=0.1,0.2,0.3,0.4,0.5,0.6,0.7,0.8,0.9$ in Step 4 we get, $f=[f 1, f 2, f 3, f 4, f 5, f 6, f 7, f 8, f 9], C=[c 1, c 2, c 3, c 4, c 5, c 6, c 7, c 8, c 9]$

Step 6 For $\mathrm{n}=0$, we obtain system of linear equations presented in (21) and solve for $\mathrm{C}$

Step 7 For different values of $n$ system of nonlinear equations $\mathrm{f}(\mathrm{c} 1, \mathrm{c} 2, \ldots, \mathrm{c} 9)=0$

Step 8 Find jacobian function, $\mathrm{J}=$ jacobian (f, C)

Step 9 Using Newton-Raphson method, $\mathrm{C}=\mathrm{C} 0$-inv $(\mathrm{J})^{*} \mathrm{f}$, where $\mathrm{C} 0$ is the initial value, using initial value computed in step 6.

Step 10 Putting C again in Step 3 and Repeat Step 3 to Step 9 (exclude Step 6) until we get correct value of C i. e. $\mathrm{f}(\mathrm{C})=0$

Step $11 \theta=1-$ transpose $(C) * P^{2} * S i 1+$ transpose $(C) * P^{2} * S i$;

End

\section{Convergence analysis of the Legendre Wavelets}

$\theta$ is the exact solution of eqns. (4) to (6). Let $\theta_{m}(X)$ be the approximate solution defined in equation (19) and let,

$\theta(X)=\sum_{m=0}^{\infty} b_{m} \psi_{1, m}(X)$ and

$\theta_{m}(X) \approx \sum_{m=0}^{M-1} b_{m} \psi_{1, m}(X)$

Thus, the error is defined as,

$$
\begin{aligned}
& E_{m}(X)=\theta(X)-\theta_{m}(X) \\
& =\sum_{m=0}^{\infty} b_{m} \psi_{1, m}(X)-\sum_{m=0}^{M-1} b_{m} \psi_{1, m}(X) \\
& =\sum_{m=M}^{\infty} b_{m} \psi_{1, m}(X) \\
& =\sum_{m=M}^{\infty}<\theta(X), \psi_{1, m}(X)>\psi_{1, m}(X) \\
& \left\|\theta(X)-\theta_{m}(X)\right\|^{2}=\sum_{m=M}^{\infty}\left|<\theta(X), \psi_{1, m}(X)>\right|^{2}
\end{aligned}
$$

The fact that $\left\|E_{m}(X)\right\|^{2}=\sum_{m=M}^{\infty}\left|<\theta(X), \psi_{1, m}(X)>\right|^{2}<\infty$ This implies that the error decays to zero.

In table 2 we compute temperature for 3, 5, 7 and 10 Legendre wavelet basis functions, we observed that error decreases as number of basis functions increases. Hence $\lim _{m \rightarrow \infty}\left\|E_{m}(X)\right\|^{2}=0$

This shows that the error decreases as the number of Legendre wavelet basis functions increases and approaches zero. 


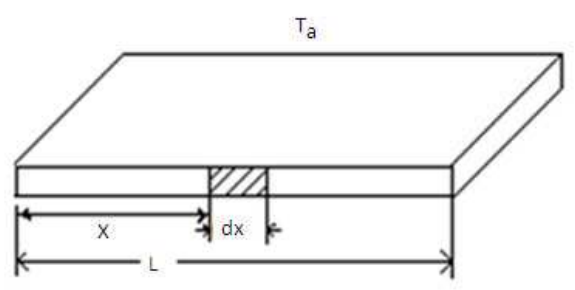

Fig. 1. Geometry of rectangular fin

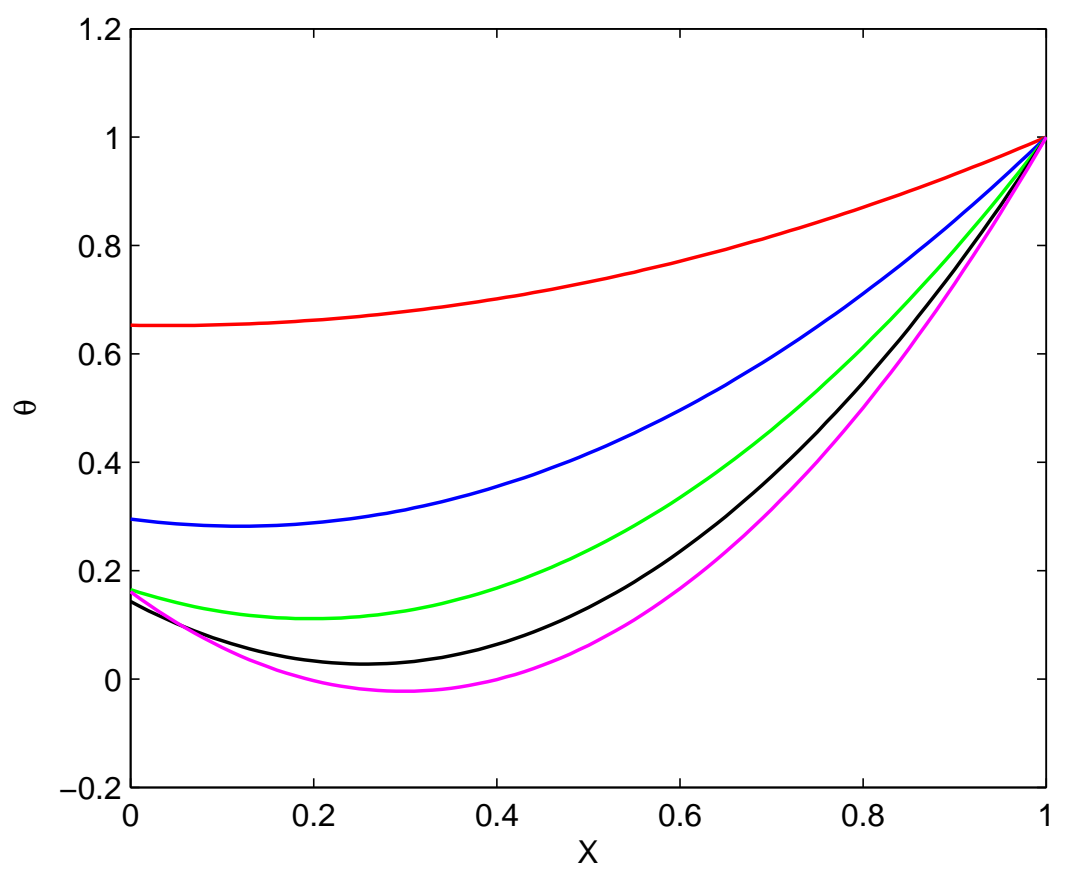

Fig. 2. WGM 3 basis functions used for $\mathrm{n}=0, \mathrm{~N}=1,2,3,4,5$ top to bottom respectively

Table 1: Comparison of WCM, WGM with Exact solution for $\mathrm{n}=0$

\begin{tabular}{lrrrrrr}
\hline Method & & & & $\theta$ & 0.6 & 0.8 \\
& $\mathrm{~N} / \mathrm{X}$ & 0.0 & 0.2 & 0.4 & 0.6 & 0.6850958012 \\
\hline Exact & 2 & 0.2658022288 & 0.2873514460 & 0.3554931896 & 0.4812762854 & 0.655095812 \\
WGM & 2 & 0.2658022293 & 0.2873514462 & 0.3554931892 & 0.4812762850 & 0.6850958012 \\
WCM & 2 & 0.2658018337 & 0.2873511951 & 0.3554930420 & 0.4812761798 & 0.6850957523 \\
Exact & 3 & 0.0993279274 & 0.1177498032 & 0.1798486648 & 0.3086588701 & 0.5519600449 \\
WGM & 3 & 0.0993279474 & 0.1177498161 & 0.1798486538 & 0.3086588612 & 0.5519600462 \\
WCM & 3 & 0.0993236495 & 0.1177472756 & 0.1798473867 & 0.3086580514 & 0.5519597814 \\
Exact & 4 & 0.0366189935 & 0.0489755216 & 0.0943841546 & 0.2034898120 & 0.4499246170 \\
WGM & 4 & 0.0366192296 & 0.0489756842 & 0.0943840448 & 0.2034897503 & 0.4499246508 \\
WCM & 4 & 0.0366084758 & 0.0489696187 & 0.0943821318 & 0.2034881699 & 0.4499250150 \\
Exact & 5 & 0.0134752822 & 0.0207934470 & 0.0506966487 & 0.1356645867 & 0.3679861444 \\
WGM & 5 & 0.0134767335 & 0.0207944713 & 0.0506960043 & 0.1356643768 & 0.3679864305 \\
WCM & 5 & 0.0134613292 & 0.0207841286 & 0.0506958553 & 0.1356613463 & 0.3679901036 \\
\hline
\end{tabular}




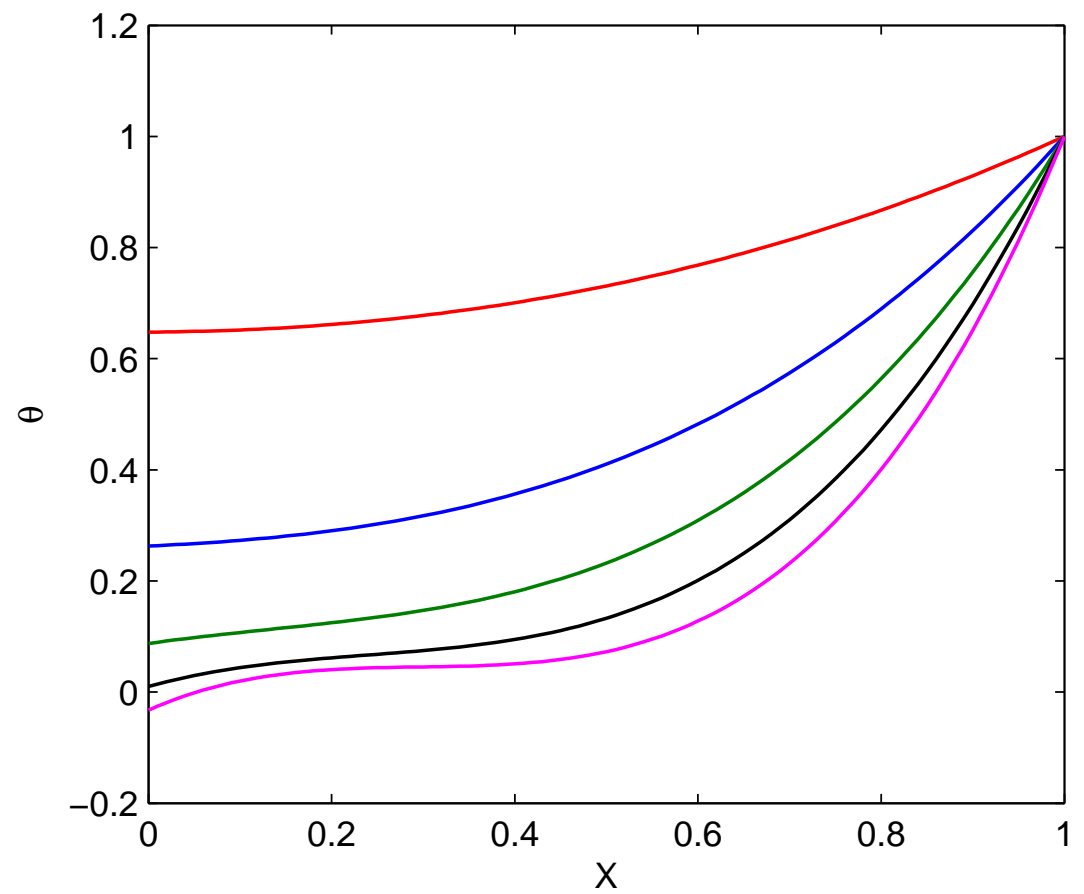

Fig. 3. WGM 4 basis functions used for $\mathrm{n}=0, \mathrm{~N}=1,2,3,4,5$ top to bottom respectively

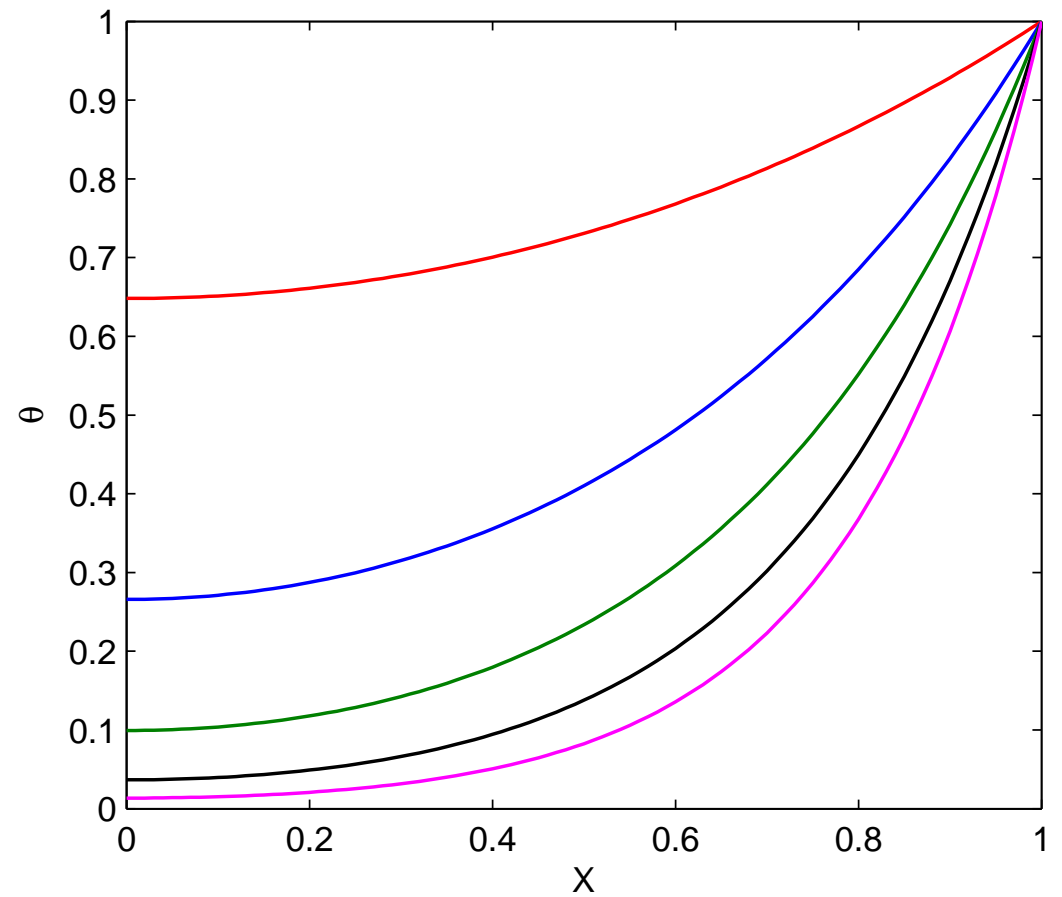

Fig. 4. WGM 10 basis functions used for $\mathrm{n}=0, \mathrm{~N}=1,2,3,4,5$ top to bottom respectively 


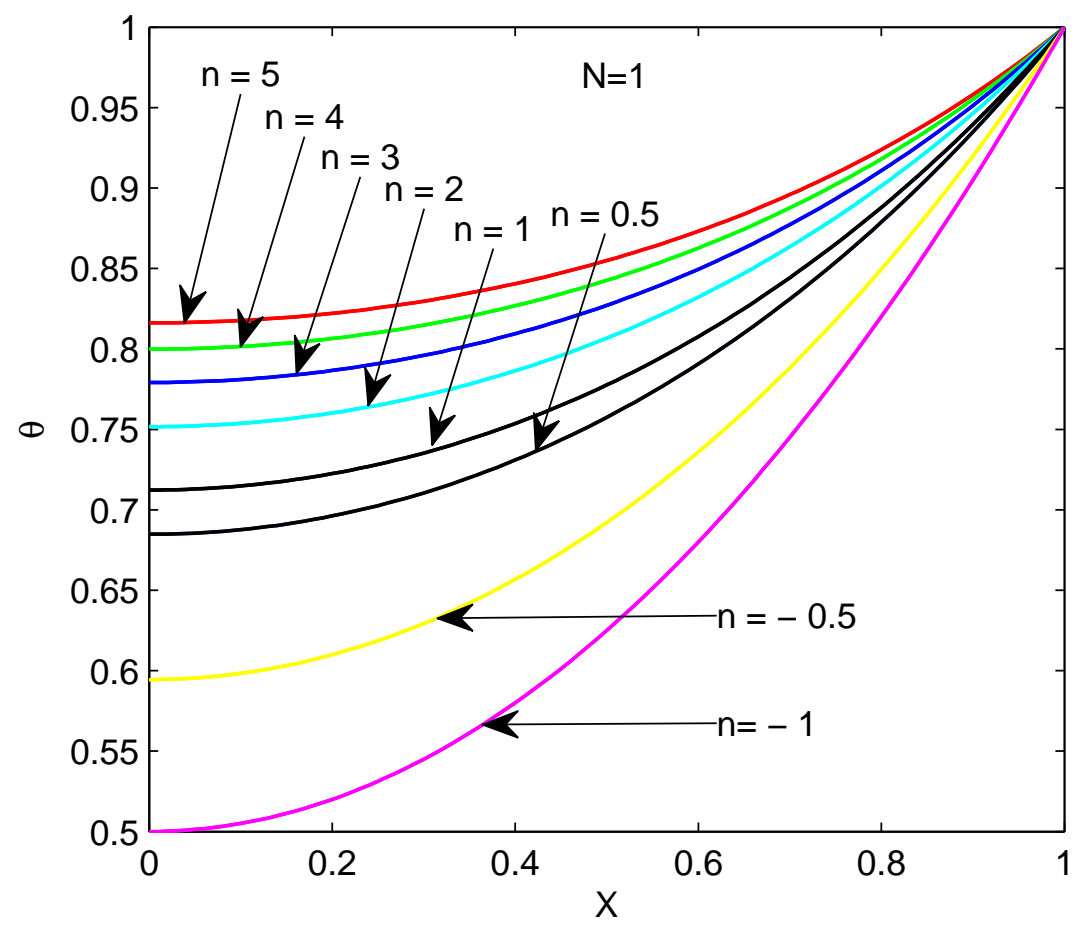

Fig. 5. Temperature in fin by WCM when 9 basis functions used

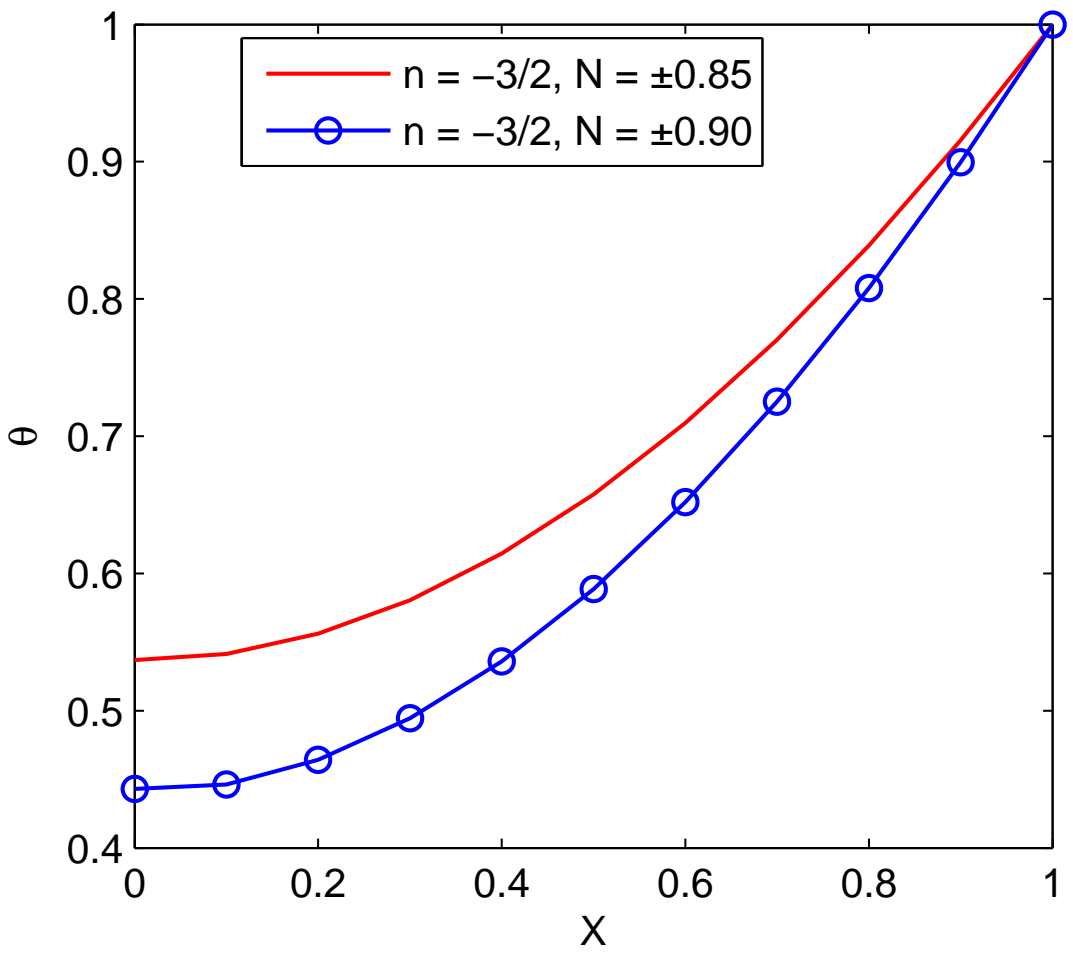

Fig. 6. Temperature distribution in fin for $\mathrm{n}=-3 / 2$ and $N= \pm 0.85, \pm 0.9$ 


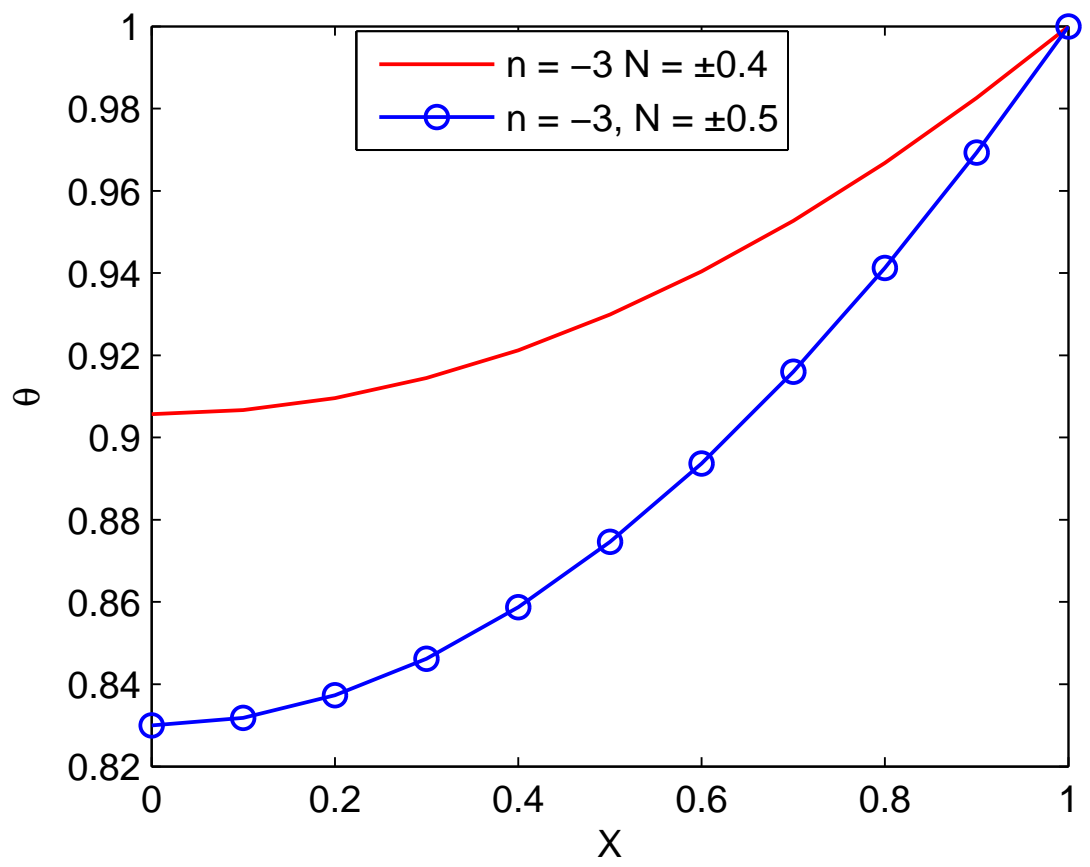

Fig. 7. Temperature distribution in fin for $\mathrm{n}=-3$ and $N= \pm 0.4, \pm 0.5$

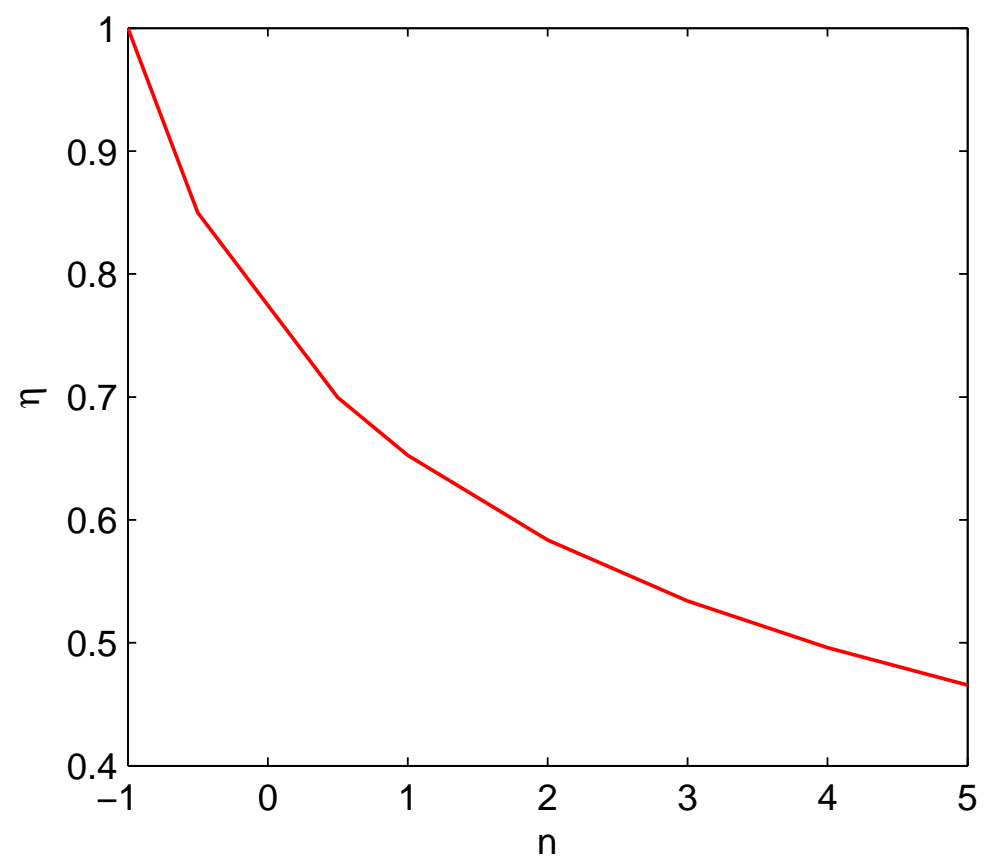

Fig. 8. Fin efficiency for $\mathrm{N}=1$ 


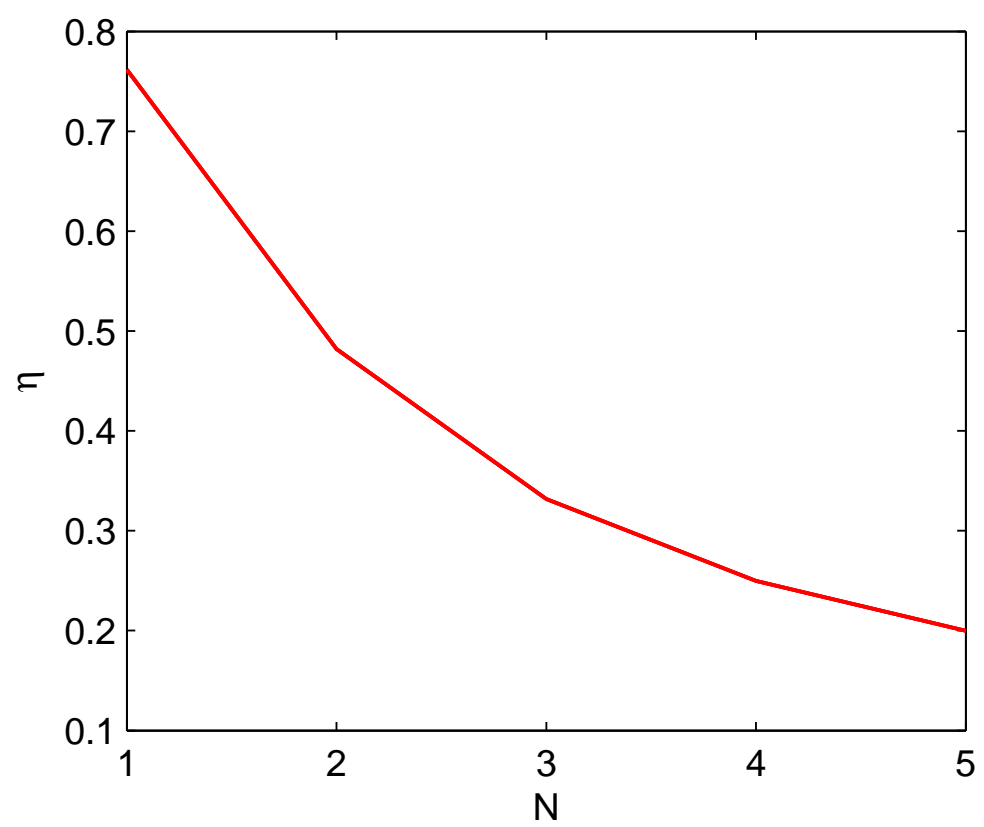

Fig. 9. Fin efficiency for $n=0$

Table 2: Comparison of present result with Numerical result and Exact result for $n=0, N=1$

\begin{tabular}{rrrrrrrr}
\hline $\mathrm{X}$ & $\begin{array}{r}\text { WGM } \\
3 \text { basis }\end{array}$ & $\begin{array}{r}\text { WGM } \\
5 \text { basis }\end{array}$ & $\begin{array}{r}\text { WGM } \\
7 \text { basis }\end{array}$ & $\begin{array}{r}\text { WGM } \\
10 \text { basis }\end{array}$ & $\begin{array}{r}\text { WCM } \\
9 \text { basis }\end{array}$ & $\begin{array}{r}\text { Numerical } \\
\text { result } \\
\text { Cortel }(2008)\end{array}$ & $\begin{array}{r}\text { Exact } \\
\text { result }\end{array}$ \\
& & & & & & \\
& functions & functions & functions & functions & functions & & \\
\hline 0.0 & 0.652817 & 0.648073 & 0.64805430 & 0.6480542737 & 0.64805431 & - & 0.6480542737 \\
0.1 & 0.653728 & 0.651300 & 0.65129725 & 0.6512972462 & 0.65129730 & 0.6512973 & 0.6512972462 \\
0.2 & 0.662151 & 0.661064 & 0.66105864 & 0.6610586204 & 0.66105869 & 0.6610587 & 0.6610586204 \\
0.3 & 0.678088 & 0.677446 & 0.67743611 & 0.6774360915 & 0.67743616 & 0.6774361 & 0.6774360915 \\
0.4 & 0.701537 & 0.700605 & 0.70059357 & 0.7005935707 & 0.70059364 & 0.7005956 & 0.7005935707 \\
0.5 & 0.732498 & 0.730772 & 0.73076284 & 0.7307628258 & 0.73076289 & 0.7307628 & 0.7307628258 \\
0.6 & 0.770973 & 0.768251 & 0.76824582 & 0.7682458010 & 0.76824586 & 0.7682457 & 0.7682458010 \\
0.7 & 0.816960 & 0.813423 & 0.81341765 & 0.8134176383 & 0.81341769 & 0.8134175 & 0.8134176383 \\
0.8 & 0.87046 & 0.866741 & 0.86673044 & 0.8667304327 & 0.86673047 & 0.8667301 & 0.8667304327 \\
0.9 & 0.931474 & 0.928733 & 0.92871778 & 0.9287177566 & 0.92871777 & 0.9287174 & 0.9287177566 \\
1.1 & 1.0 & 1.0 & 1.0 & 1.0 & 1.0 & 0.9999995 & 1.0 \\
\hline
\end{tabular}

Table 3: Present solution by Wavelet Collocation Method for $\mathrm{N}=1$

\begin{tabular}{lrrrrrr}
\hline $\mathrm{n} / \mathrm{X}$ & 0.0 & 0.2 & 0.4 & 0.6 & 0.8 & 1.0 \\
-1 & 0.50000 & 0.520000 & 0.580000 & 0.680000 & 0.820000 & 1.0 \\
-0.5 & 0.594446 & 0.609899 & 0.656656 & 0.735887 & 0.849486 & 1.0 \\
0.5 & 0.684781 & 0.696161 & 0.730875 & 0.790695 & 0.878779 & 1.0 \\
1 & 0.712256 & 0.722451 & 0.753627 & 0.807647 & 0.887931 & 1.0 \\
2 & 0.751622 & 0.760163 & 0.786380 & 0.832191 & 0.901271 & 1.0 \\
3 & 0.779145 & 0.786562 & 0.809396 & 0.849546 & 0.910778 & 1.0 \\
4 & 0.799839 & 0.806431 & 0.826770 & 0.862712 & 0.918037 & 1.0 \\
5 & 0.816146 & 0.822101 & 0.840505 & 0.873163 & 0.923832 & 1.0 \\
\hline
\end{tabular}


Table 4: Comparison with other results at single point $\mathrm{X}=0.0, \mathrm{~N}=1$

\begin{tabular}{lrrrrr}
\hline $\mathrm{n}$ & WCM & Num.Sol & DTM & ADM & Exact Sol. \\
& & & Moradi(2012) & Chang(2005) & \\
-1 & 0.5 & 0.495008 & 0.5 & 0.5 & 0.5 \\
-0.5 & 0.5944462738 & 0.59186 & 0.594447 & 0.594458 & 0.594446 \\
0.5 & 0.6847812223 & 0.68466 & 0.684781 & 0.684779 & 0.684781 \\
1 & 0.7122565534 & 0.712166 & 0.712258 & 0.712263 & 0.712256 \\
2 & 0.7516220641 & 0.751568 & 0.751635 & 0.75166 & 0.751622 \\
3 & 0.7791447258 & 0.77911 & 0.779177 & 0.779227 & 0.779145 \\
4 & 0.7998388152 & 0.79982 & 0.799894 & 0.79994 & 0.79984 \\
5 & 0.8161462380 & 0.816135 & 0.816226 & 0.816268 & 0.816149 \\
\hline
\end{tabular}

\section{Result and Discussion}

Now we consider the linear equation (21). Taking the actual physiological data from Chang (2005), abbasbandy and shivainian (2010) and incorporating it in equation (19), we evaluate the temperature $\theta$ when three, five, seven and ten basis functions are taken. While considering the equation (19), we find temperature $\theta$ when nine collocation points are taken.

In Fig. 1, geometry of the fin is given. Figs. 2, 3 and 4 represent solution obtained by Wavelet Galerkin Method using three, four and ten Legendre wavelet basis functions. When we compare Figs. 2, 3 and 4, we observe that the curves for $N=4$ and 5 are opposite at $x=0$, numerical data shown in Table 1 and Table 2. From Figs. 2, 3 and 4 has it been observed that the curves for $N=1,2$ and 3 are same. For $N=4$ and 5 curves change rapidly. This is clear that for higher values of $N(N=4,5)$ higher order Legendre wavelet basis functions are required. Numerical data is given in table 1 and table 2. In Fig. 4 we use ten Legendre wavelet basis functions. The result thus obtained is correct up to ten decimal places. In table 1 result is given for $n=0, N=2,3,4,5$ calculated by WCM, WGM and Exact method. We observe that WGM is better in comparison to WCM. Accuracy decreases as value of $N$ increases

From table 2 it is clear that the accuracy increases as we increase number of Legendre wavelet basis functions. It also indicates that the method converges. When we compare our result with Cortell (2008), we find that our result is better. It has been observed that the Wavelet Galerkin Method and Wavelet collocation method give correct result up to ten decimal places. In table 2 when we compare second, third, fourth and fifth columns with exact result, we observe that the accuracy increases up to ten decimal places. Temperature distribution for $N=1$ and different values of $\mathrm{n}$ is given in table 3 . It has been observed that as value of $\mathrm{n}$ increases temperature in fin increases.

In nonlinear case problem solved by WCM, temperature distribution is presented in Fig.5. A comparative study of present result with other results is shown in table 4 . In table 4 we observe that our result calculated by WCM is better in comparison to DTM Moradi (2005), Numerical solution Moradi (2005), ADM Chang (2010) and near to exact one.

The exact solution obtained by Abbasbandy and Shivanian (2010) is in implicit form and in a set of subintervals while the present method provides single solution for all existing values. Abbasbandy and Shivanian (2010) plot dual solution, while our observation is that in any physical problem one solution exists not dual. In Figs. 6 and 7 we plot temperature distribution in fin for $n=-3 / 2, N= \pm 0.85, \pm 0.90$ and $n=-3, N= \pm 0.4, \pm 0.5$ respectively. By wavelet collocation method we get unique solution; this is the merit of our work. It is clear from figs. 6 and 7 , temperature between two nearest points increases or decreases slowly but not rapidly.

Most important part of any system is its efficiency. The fin efficiency is the ratio of total heat transfer rate to that of fin at base temperature. Thus, we get

$\eta=\frac{\left.k A_{c} \frac{d T}{d X}\right|_{X=L}}{\operatorname{Ph}\left(T_{b}-T_{a}\right) L}$

In dimensionless form, we obtain

$\eta=\frac{1}{N^{2}} \theta^{\prime}(1)$, for $N \neq 0, N=1,2,3,4,5$ 
For the case $n=-1$ indicates an uniform local heat flux over the whole fin surface and induces the result of $\eta=1$, which is clear from Fig.8. It has been observed that the value of $\eta$ decreases as $n>-1$. From Fig. 8 and 9 , we observe that the efficiency decreases as the value of $\mathrm{n}$ or $\mathrm{N}$ increases.

\section{Conclusion}

The present method has been used to analyze the heat conduction for a fin with heat transfer coefficient varying as a power law function of temperature. It is shown that method is reliable and gives sufficient accuracy. WCM is applied in both cases linear as well as in nonlinear; but WGM is very difficult to apply in nonlinear case. These methods may be applied in different difficult linear and nonlinear engineering problems. The obtained solution for temperature distribution offers many advantages over the other methods. Some of the advantages are given below: 1. The present method shows excellence performance for linear and highly nonlinear boundary value problem. Simple applicability and fast convergence of the Legendre wavelet provide a solid foundation for using these functions in context of numerical approximations of boundary value problems.

2. The exact solution obtained by Abbasbandy and Shivanian (2010) is in implicit form and provides solution in a number of subintervals while the present method provides single solution for all existing values.

3. The method proposed by Abbasbandy and Shivanian (2010) for $n=-3, N= \pm 0.4$ and $n=-3 / 2, N= \pm 0.9$ provides dual solution while the present method gives a single solution. The justification of unique solution gets confirmed from Figs. 5 and 6.

4. The method can be applied in multidimensional linear and nonlinear heat transfer problem..

\section{Acknowledgements}

Authors are grateful to Prof. Umesh Singh coordinator DST- Centre for interdisciplinary Mathematical Sciences Banaras Hindu University Varanasi, India for providing necessary facilities.

\section{References}

[1] I.N. Dul'kin, G.I. Garas'ko, "Analysis of the 1-D heat conduction problem for a single fin with temperature dependent heat transfer coefficient: Part I Extended inverse and direct solutions", International Journal of Heat and Mass Transfer 51, (2008), pp.3309-3324.

[2] I.N. Dul'kin, G.I. Garas'ko, "Analytical solutions of the 1-D heat", Int. J. of Heat and Mass Transfer, Vol.45(2), (2002), pp.1895-1903.

[3] A. Moradi, "Analytical solution for fin with temperature dependent heat transfer coefficient", Int. J. of Eng. and App. Science, Vol.3, issue 2, (2011), pp.1-12.

[4] Min-Hsing Chang, "A decomposition solution for fins with temperature dependent surface heat flux", Int. J. of Heat and Mass Transfer, 48 (2005), pp.1819-1824.

[5] M.S.H. Chowdhury, I.Hashim and O. Abdulaziz, "Comparison of homotopy analysis method and homotopy-perturbation method for purely nonlinear fin-type problems", Communications in Nonlinear Science and Numerical Simulation, 14, (2009), pp.371-378.

[6] Rafael Cortell, "A numerical analysis to the non-linear fin problem", J. of Zhejiang University Science, A 9 (5), (2008), pp.648-653.

[7] S. Abbasbandy and E. Shivanian, "Exact analytical solution of a nonlinear equation arising in heat transfer", Physics Letters A, 374 (2010), pp.567-574.

[8] Chang M.H., "A decomposition solution for fins with temperature dependent surface heat flux", Int. J. Heat Mass Transfer", 48 (2005), pp.1819-1824.

[9] K. N. Rai and K.D. Rai, "A Numerical Technique for the solution of Transport Problem", Proceeding, Fourth National Conference on Thermal Systems Dept. of Mech. Eng. IT-BHU, (2003), pp.268-277.

[10] F. Khani, M. Ahmadzadeh Raji, H. Hamedi Nejad, "Analytical solution and efficiency of the nonlinear fin problem with temperature-dependent thermal conductivity and heat transfer coefficient", Commun Nolinear Sci Numer Simulat, 14 (2009), pp.3327-3338. 
[11] Sin Kim and Cheng-Hung Huang, "A series solution of the non-linear fin problem with temperature dependent thermal conductivity and heat transfer coefficient", J. Phys. D: Appl. Phys., 40, (2007), pp.2979-2987.

[12] Ebrahim Momoniat, "A comparison of two formulations of the fin efficiency for straight fins", Acta Mech. Sin., 28 (2), (2012), pp.444-449.

[13] Ching - Huang Chiu and Cha'o- Kuang Chen, "A decomposition method for solving the convective longitudinal fins with variable thermal conductivity", Int. J. of Heat and Mass Transfer, 45 (2002), pp.2067-2075.

[14] A Rajabi, "Homotopy perturbation method for fin efficiency of convective straight fins with temperature-dependent thermal conductivity", Physics Letters A, 364, (2007), pp.33-37.

[15] Cihat Arslanturk, "A decomposition method for fin efficiency of convective straight fins with temperature-dependent thermal conductivity", Int. Commun. in Heat and Mass Transfer, 32(2005), pp.831-841.

[16] G. Domairry and M. Fazeli, "Homotopy analysis method to determine the fin efficiency of convective straight fins with temperature-dependent thermal conductivity", Commun. in nonlinear Sci. and Nonlinear Simulation,14 (2009), pp.489-499.

[17] D.D. Ganji, M.J. Hosseini and J. Shayegh, Some nonlinear heat transfer equations solved by three approximate methods, Int. Commun. in Heat and Mass Transfer, 34 (2007), pp.1003-1016.

[18] M. Razzaghi and S. Yousefi, "The Legendre wavelets operational matrix of integration", Int.J. of Systems Science, Vol.32, No.4, (2001), pp.495-502.

[19] F. Mohammadi, M.M. Hosseini and syed Tauseef Mohyud-Din, "Legendre Wavelet Galerkin method for solving ordinary differential equations with non-analytic solution", Int. J. of Sys. Sci., Vol. 42, No. 4, (2011), pp.579-585.

[20] M.W. Frazier, "An Introduction to Wavelets Through Linear Algebra", Springer-Verlag New York Berlin Heidelberg, SPIN 10557627, (1999), pp.470-480.

\section{Nomenclature}

Ac - cross section area of the fin $\left(m^{2}\right)$

$h$ - heat transfer coefficient $\left(W m^{-2} K^{-1}\right)$

$h_{b}$ - heat transfer coefficient at fin base

$k \quad$ - thermal conductivity of fin material $\left(W m^{-1} K^{-1}\right)$

$k_{a}$ - thermal conductivity of fin material at ambient temperature $\left(W m^{-1} K^{-1}\right)$

$L \quad$ - length of fin $(\mathrm{m})$

$P \quad$ - circumferential parameter(m)

$T$ - local temperature on the fin $\operatorname{surface}(\mathrm{K})$

$T_{a}$ - environment (ambient) temperature $(\mathrm{K})$

$T_{b}$ - base temperature of the fin $(\mathrm{K})$

$N$ - convective- conduction dimensionless fin parameter

$x$ - dimensional space coordinate

$X$ - dimensionless space coordinate

$\theta \quad$ - dimensionless temperature

$\eta \quad$ - fin efficiency 